\title{
Flexible Polyaniline/Poly(methyl methacrylate) Composite Fibers via Electrospinning and In Situ Polymerization for Ammonia Gas Sensing and Strain Sensing
}

\author{
Xian-Sheng Jia, ${ }^{1}$ Cheng-Chun Tang, ${ }^{1,2}$ Xu Yan, ${ }^{1}$ Gui-Feng Yu, ${ }^{1}$ Jin-Tao Li, ${ }^{1}$ \\ Hong-Di Zhang, ${ }^{1}$ Jun-Jie Li, ${ }^{2}$ Chang-Zhi Gu, ${ }^{2}$ and Yun-Ze Long ${ }^{1,3}$ \\ ${ }^{1}$ Collaborative Innovation Center for Nanomaterials \& Devices, College of Physics, Qingdao University, Qingdao 266071, China \\ ${ }^{2}$ Laboratory of Microfabrication, Institute of Physics, Chinese Academy of Sciences, Beijing 100190, China \\ ${ }^{3}$ Industrial Research Institute of Nonwovens \& Technical Textiles, College of Textiles \& Clothing, Qingdao University, \\ Qingdao 266071, China
}

Correspondence should be addressed to Chang-Zhi Gu; czgu@iphy.ac.cn and Yun-Ze Long; yunze.long@163.com

Received 26 July 2016; Revised 10 November 2016; Accepted 24 November 2016

Academic Editor: Russell E. Gorga

Copyright (C) 2016 Xian-Sheng Jia et al. This is an open access article distributed under the Creative Commons Attribution License, which permits unrestricted use, distribution, and reproduction in any medium, provided the original work is properly cited.

\begin{abstract}
Conducting polyaniline (PANI) was in situ polymerized at the surface of electrospun poly(methyl methacrylate) (PMMA) fibers to obtain flexible composite fibers. The electrical conductivity of an individual PANI/PMMA composite fiber was estimated to be $2.0 \times 10^{-1} \mathrm{~S} \mathrm{~cm}^{-1}$ at room temperature. The ammonia sensing properties of the samples were tested by impedance analysis. The PANI/PMMA fibers could obviously respond to low concentration of ammonia at ppb level and could respond to relatively high concentration of ammonia at $10 \mathrm{ppm}$ level quickly. In addition, the sensitivity exhibited a good linear relationship to the ammonia concentration. Particularly, the flexible PANI/PMMA fibers showed a reversible change in electrical resistance with repeated cycles of bending and relaxing, and the electrical resistance decreased with the increase of curvature. These results indicate that the flexible PANI/PMMA composite fibers may be used in toxic ammonia gas detection, strain sensing, and flexible electronic devices.
\end{abstract}

\section{Introduction}

Nowadays, ultrathin fibers and fibrous membranes are well used in lithium-ion batteries [1,2], supercapacitors [3], tissue engineering [4], drug delivery [5], sensors [6], and flexible electronic devices [7] because of high surface area, low weight, and good flexibility. There are many methods to fabricate ultrathin fibers such as hydrothermal method, carbothermal reduction, sol-gel method, solid-state chemical reaction, chemical vapor deposition, and electrospinning. Compared with other methods, electrospinning is a simple and convenient way to fabricate continuous long fibers. Various organic and inorganic materials have been prepared into ultrathin fibers by solvent electrospinning or melt electrospinning [8]. The morphology of electrospun fibers also could be beaded string [9], belt [10], porous structure [11], or totally hollow microtube $[12,13]$.
It is crucial to choose suitable materials for the fabrication of high-performance sensors. Using nanostructured materials to fabricate high sensitive sensors has attracted extensive concern for the high surface to volume ratio [14]. Another increasing tendency of developing smart sensors is to use flexible materials, which can overcome the structural restrictions of conventional sensors [15-18]. Polyaniline (PANI) is a promising conducting polymer for application in electronics because of its environmental stability, low cost, and easy processability [19]. PANI used as gas or strain sensor has drawn much attention recently [20-23]. But PANI has a fairly rigid backbone due to high aromaticity and it is available only in relatively low molecular weight forms, so that its solution elasticity is generally insufficient to be electrospun directly into fibers [24, 25]. And this property of PNAI limits its applications. Combining PANI with other polymers to obtain conductive fibers is an effective way. For instance, composite 


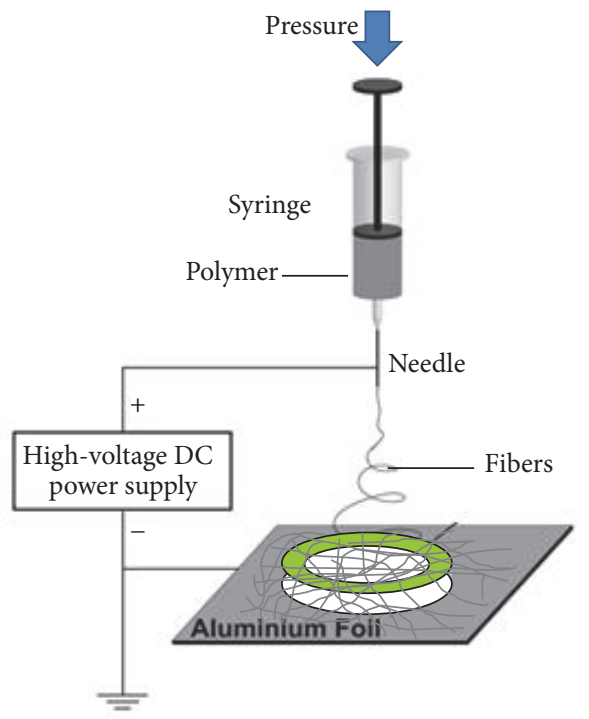

(a)

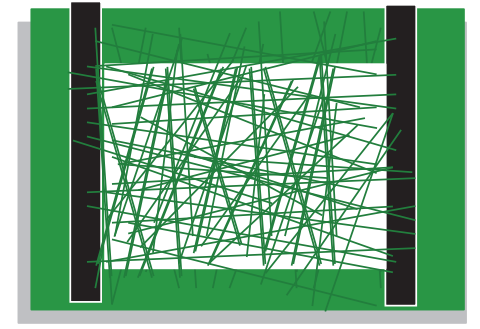

(b)

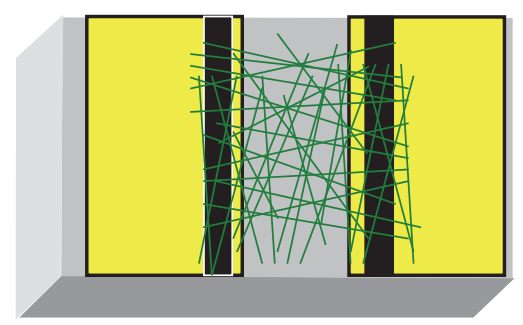

(c)

FIgure 1: (a) Schematic illustration of electrospinning setup for fabrication of PMMA fibers; (b) schematic illustration of PANI/PMMA composite fibers after in situ polymerization of PANI; (c) schematic illustration of a device based on the composite fibers for electrical and gas sensing measurements.

PANI fibers could be fabricated by direct electrospinning of a precursor solution containing PANI $[26,27]$ or by in situ polymerization of PANI on the surface of electrospun polymer fibers [28]. Here, we fabricated PANI/PMMA composite fibers by in situ polymerization method. The PANI/PMMA composite fibers combined the good properties of PMMA fibers (e.g., high surface area and flexibility [29]) and the high electrical conductivity of PANI, showing excellent strain and gas sensing properties.

\section{Experimental}

2.1. Materials and Instruments. PMMA (average degree of polymerization is 43,982 ) was purchased from Alfa Aesar, Tianjin. N,N-dimethyl formamide (DMF, AR), tetrahydrofuran (THF, AR), aniline, ammonium peroxodisulfate (APS), and 5-sulfosalicylic acid dihydrate (SSA) were all purchased from Sinopharm Chemical Reagent, Beijing. All the materials were used without further purification. A high-voltage DC power supply (DW-P303-1ACFO, Tianjin Dongwen) and a magnetic stirrer (HJ-4A, JinTan Youyi Instrument Institute) were employed in the electrospinning process.
2.2. Preparation of Electrospun PMMA Fibers. $2.0 \mathrm{~g}$ PMMA, $10.0 \mathrm{~g}$ DMF, and $10.0 \mathrm{~g}$ THF were mixed to form the electrospinning precursor solution. The solution was stirred for $2 \mathrm{~h}$ at room temperature. As shown in Figure 1(a), a syringe with a stainless steel needle was connected to the positive electrode of the high-voltage power supply. Aluminum foil $\left(5 \times 5 \mathrm{~cm}^{2}\right)$ on a hollow cylinder $\left(3 \times 3 \times 1 \mathrm{~cm}^{3}\right)$ was connected to the ground as a collector. A high DC voltage of $12 \mathrm{kV}$ was applied in the process of electrostatic spinning. The distance to collector was $10 \mathrm{~cm}$ and the flow rate was $35 \mu \mathrm{L} \mathrm{min}^{-1}$. The RH was $65 \%$. A spinning time of $3 \mathrm{~min}$ was used to obtain a fibrous membrane.

2.3. Deposition of PANI on the Surface of PMMA Fibers. The electrospun PMMA fibrous mat was then transformed to a piece of polyethylene (PE) slice with a thickness of $0.6 \mathrm{~mm}$ and a hole sized by $1 \times 2 \mathrm{~cm}^{2}$. The mat covered the hole and was fixed to the PE substrate by double-sided adhesive tape, as shown in Figure 1(b). To grow PANI nanostructures on the surface of electrospun PMMA fibers by in situ polymerization, the reaction solution was prepared as follows. Firstly, $0.01 \mathrm{~mol}$ sulfosalicylic acid (SSA) dehydrate was dissolved 


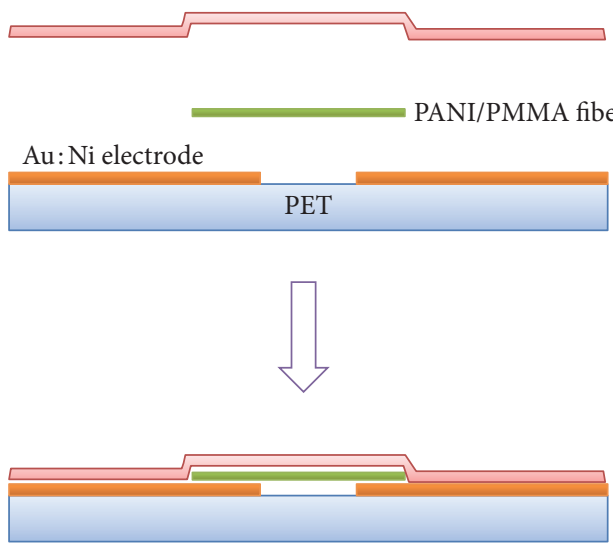

(a)
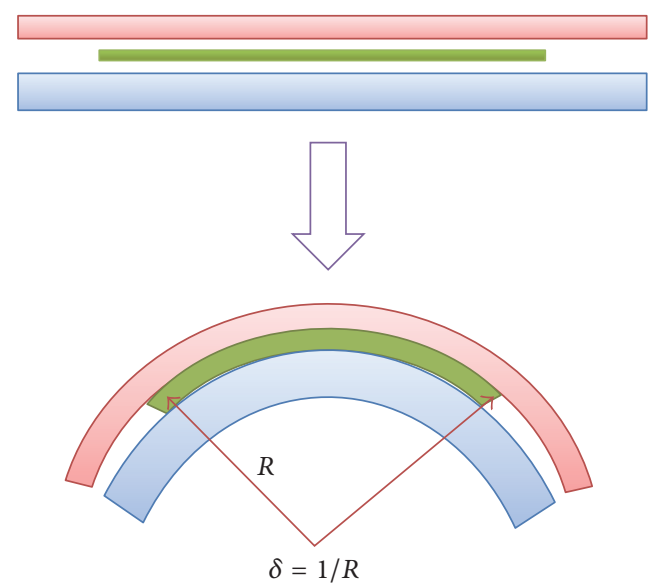

(b)

FIGURE 2: (a) Schematic illustration of fabrication of strain sensor; (b) schematic illustration of strain sensing test.

thoroughly in $50 \mathrm{~mL}$ deionized water. Then, $0.02 \mathrm{~mol}$ aniline was added and stirred to form a stable solution named $\mathrm{A}$. Secondly, $0.02 \mathrm{~mol}$ APS was dissolved and stirred in $50 \mathrm{~mL}$ deionized water to form solution B. Both solution A and solution $\mathrm{B}$ were kept in a refrigerator with the temperature of $5^{\circ} \mathrm{C}$ for $1 \mathrm{~h}$. Then, solution $\mathrm{A}$ and solution $\mathrm{B}$ were mixed together and the PMMA mat pasted on the PE substrate was immersed in the mixed solution and kept steady at $5^{\circ} \mathrm{C}$ for $6.5 \mathrm{~h}$. As PANI grew from chemical oxidative polymerization process of aniline, the solution gradually turned green, as well as the fibers and the PE substrate deposited with PANI (Figure 1(b)). At last, the fibrous mat was taken out, washed with water for 3 times, dried in air. As shown in Figure 1(c), the PANI/PMMA fibrous web was fixed on gold electrodes by carbon paster.

2.4. Characterization of Morphology and Structures. The resultant PANI/PMMA fibers were characterized by Raman spectrum analysis (Jobin Yvon SA, excitation wavelength $\sim 532.16 \mathrm{~nm}$ ) and a scanning electron microscope (SEM, Hitachi TM-1000).

2.5. Electrical and Gas Sensing Measurement. As shown in Figure 1(c), the fibrous mat (removed from the PE slice) was fixed on the $\mathrm{Au} / \mathrm{Ni}$ electrodes by conductive tapes. The electrical and $\mathrm{NH}_{3}$ sensing properties of the sample were measured by a Keithley 6517 high-resistance meter and a home-made gas sensing system under constant temperature $\left(18^{\circ} \mathrm{C}\right)$. The electrical data was recorded automatically by a computer.

2.6. Fabrication of Strain Sensor. The as-prepared composite fibers were also tested as a strain sensor. As shown in Figure 2(a), a piece of fibrous mat was placed on a plastic poly(ethylene terephthalate) (PET) substrate with prepatterned $\mathrm{Au} / \mathrm{Ni}$ electrodes. Then, the PDMS (Dow Corning Sylgard 184; ratio of base to crosslinker, $10: 1$ by mass) thin layer was mixed, degassed, and poured against the surface of the membranes to package the device. To measure resistance change of this sensor under bending, the device was placed on a curved surface (e.g., surface of a cylinder) with bending curvature of $\delta=1 / r$ ( $r$ is the semidiameter of the cylinder), as shown in Figure 2(b). In the measurement process, a fixed current $(0.2 \mu \mathrm{A})$ was applied, and the voltage was recorded automatically by the computer cycles of bending and relaxing.

\section{Results and Discussion}

3.1. Raman Spectrum Analysis. Figure 3 shows the Raman spectra of the as-prepared samples. The Raman spectrum of PANI/PMMA composite fibers is matched to pure PANI Raman shift peaks. For example, representative peaks at $1179 \mathrm{~cm}^{-1}$ (C-H bending of the quinoid ring), $1251 \mathrm{~cm}^{-1}$ (C-H bending of the benzenoid ring), $1339 \mathrm{~cm}^{-1}(\mathrm{C}-\mathrm{N}$ stretching), and $1509 \mathrm{~cm}^{-1}$ and $1593 \mathrm{~cm}^{-1}$ (C-C stretching of the benzene ring) can be observed in Raman spectrum of both PANI powder and PANI/PMMA composite fibers [30]. Compared with Raman shift peaks of electrospun PMMA fiber, we can infer that the slightly higher peaks $606 \mathrm{~cm}^{-1}$ and $810 \mathrm{~cm}^{-1}$ of PANI/PMMA fibers come from PMMA. The peak of $1729 \mathrm{~cm}^{-1}$ that lies on the same position of PMMA spectrum is also scattered from PMMA. On the other hand, the $1451 \mathrm{~cm}^{-1}$ peak is notable, while being nearly undetectable in the spectrum of PANI/PMMA fibers. In addition, the laser jet was focused on the flat area of the composite fiber. As a result, we can conclude from the Raman spectra that PANI was in situ polymerized at the surface of PMMA fibers.

3.2. SEM Characterization. PANI usually shows different colors such as blue, violet, and black when doped with different acids. Namely, when oxidated to three different relatively stable structures of leucoemeraldine (LEB), emeraldine (EB), and pernigraniline (PNG), PANI shows green (LEB and $\mathrm{EB}$ ) and purple or black (PNG) and the green EB are the most conductive state of PANI. The composite fibers obtained here are well conductive EB structure with dark green color as a 


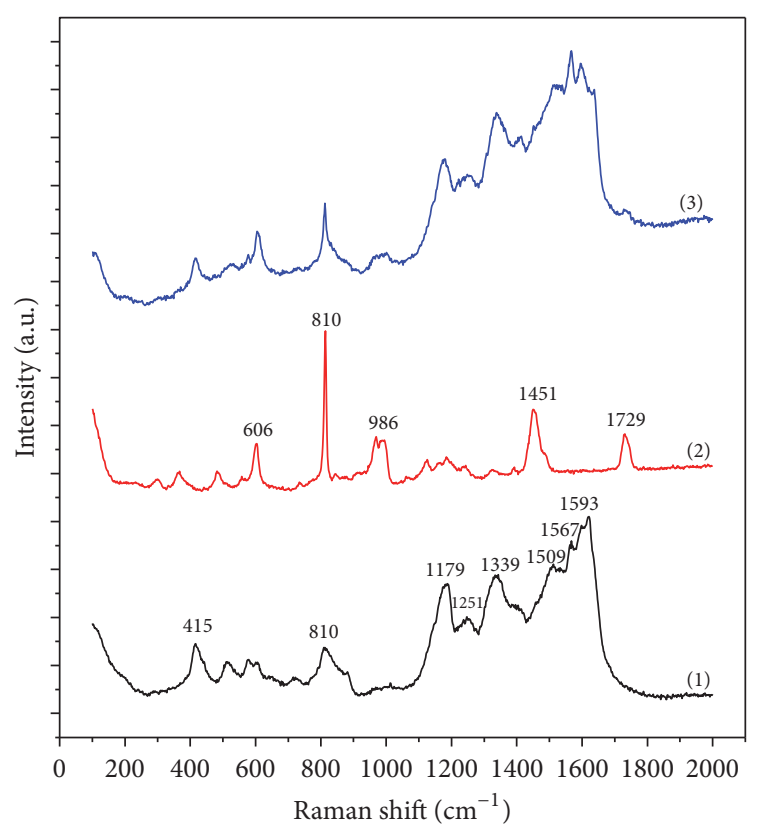

FIgURE 3: Raman spectrum of the (1) PANI powder, (2) electrospun PMMA fibers, and (3) PANI/PMMA composite fibers.

result of SSA doping and half oxidation of APS to aniline molar ratio of 1 to 1 . Figure 4 shows the morphologies and microstructures of PMMA fibers (Figure 4(a)) and PANI/ PMMA composite fibers (Figure 4(b)). After in situ polymerization of PANI, the areal density of fibrous mat was increased from $0.140 \mathrm{mg} \mathrm{cm}^{-2}$ to $0.144 \mathrm{mg} \mathrm{cm}^{-2}$. The surface of composite fibers was rough because the PANI nanostructures polymerized at the surface of the PMMA fibers. Figures 4(c) and 4(d) show the enlarged SEM pictures of the composite fibers. The average fiber diameter is $8.76 \mu \mathrm{m}$ for PMMA fibers and $9.25 \mu \mathrm{m}$ for PANI/PMMA fibers separately before and after in situ polymerization of PANI, indicating that the average thickness of PANI coating layer is about $0.49 \mu \mathrm{m}$.

\subsection{Electrical Conductivity Characterization. As shown in} Figure 5(b), a linear relationship of the current and the applied voltage was observed for the PANI/PMMA fibrous mat. The resistance of the PANI/PMMA mat calculated from the $I-V$ curve was about $2.273 \mathrm{M} \Omega$, and then the calculated room-temperature conductivity was about $2.0 \times 10^{-1} \mathrm{~S} \mathrm{~cm}^{-1}$. For comparison, the conductivity of the PMMA fibrous mat was very low, about $4.3 \times 10^{-8} \mathrm{~S} \mathrm{~cm}^{-1}$. So, the coated PANI on the surface of PMMA fibers has enhanced the electrical conductivity of the composite fibers significantly. Table 1 summaries the average diameter, areal density, and conductivity of the PMMA fibers before and after polymerization of PANI.

3.4. $\mathrm{NH}_{3}$ Gas Sensing Characterization. To test the ammonia sensing property of the composite fibers, the sample was put inside a chamber connected to a pump and the resistance signal was measured by a Keithley 6517 high-resistance meter with $I-V$ scanning mode. The scanning voltage was set from
3.72 to $4.00 \mathrm{~V}$ with a step of $0.001 \mathrm{~V}$ and a time delay of $4 \mathrm{~s}$. Thus, 280 resistance test points and $1120 \mathrm{~s}$ scanning time were obtained with the "bias voltage" varied in the range of $0.28 \mathrm{~V}$. As a result, there was a little decrease of resistance at beginning caused by the increase of "bias voltage," and then the resistance increased due to the ammonia. An ultralow concentration of $80 \mathrm{ppb}(0.08 \mathrm{ppm}) \mathrm{NH}_{3}$ gas was injected to the chamber, and the response line was plotted in Figure 6(a). With time going on, the resistance of sample first increased dramatically, then slowed down, and finally reached to a platform within about $1000 \mathrm{~s}$ by $3.8 \%$. When exposed to $400 \mathrm{ppb}$ $(0.4 \mathrm{ppm})$ ammonia, the resistance of the fibers coated by PANI was increased by $17 \%$ (Figure 6(a)). For ammonia sensitivity measurement at ppm level, a reaction time of $20 \mathrm{~min}$ was used for every test. Here, the relative change of resistance $\left(\Delta R / R_{0}, \Delta R=R-R_{0}\right)$ is defined as sensitivity. As shown in Figure 6(b), the sensitivity of the composite fibers at different concentrations of ammonia is nearly linear. The detectability to low concentration of ammonia of the PANI/PMMA fibers could be ascribed to high surface area, which provides numerous response sites.

The reversible responses of PMMA/PANI fibers at different concentrations were also tested. As shown in Figure 7, when the given concentration of $\mathrm{NH}_{3}$ was pumped into the container, the resistance of sample increased quickly. For example, the relative resistance change was more than $130 \%$ when exposed to $80 \mathrm{ppm}$ concentration of $\mathrm{NH}_{3}$. When the $\mathrm{NH}_{3}$ was pumped out, the decline of resistance was also fast. The platform in the middle of curve represents the stable process. The platform of sensitivity line (or peak height) is not uniform, which may be caused by the instability of gas flow and transport inside the chamber.

The ammonia sensing mechanism can be explained from the conducting mechanism of PANI [31]. As we know, the delocalization of $\mathrm{sp}^{3}$ electronic state is enhanced by the doping of proton, which makes the PANI conjugated molecule chain more conductive because the amount of polaron is increased. And doping of weak organic proton acid (5sulfosalicylic acid) makes the PANI structurally and electrically more stable. For PANI, the current carrier is polaron. The ammonia molecules can be absorbed by the nanostructured PANI on the surface, and then PANI in the form of emeraldine salt (ES) is reduced to emeraldine base (EB). These dedoping processes on the PANI chains can result in a decrease of the current carriers and an increase of electrical resistance. When replacing ammonia with air, the ammonia concentration around PANI decreases, more $\mathrm{NH}_{3}$ molecules on the surface of PANI are released, and then more current carriers are freed, so that the electrical conductance increases.

3.5. Strain Sensing Characterization. The PANI/PMMA fibers have excellent gas sensing property. Furthermore, the strain sensing property of the flexible composite fibers was also tested. Figure 8 (a) shows the $I-V$ curves of PANI/PMMA fibers bended at different curvatures. The $I-V$ characteristics have obvious differences when bended at different degrees. With increasing curvature, the resistance of PANI/PMMA fibers decreased gradually as shown in Figure 8(b). The decrease of resistance could be up to $35 \%$ when the bending 
TABLE 1: Average diameter, areal density, and conductivity of PMMA fibers before and after polymerization of PANI.

\begin{tabular}{lccc}
\hline & Average diameter $(\mu \mathrm{m})$ & Areal density $\left(\mathrm{mg} \mathrm{cm}^{-2}\right)$ & Conductivity $\left(\mathrm{S} \mathrm{cm}^{-1}\right)$ \\
\hline Before polymerization & 8.76 & 0.140 & $4.3 \times 10^{-8}$ \\
After polymerization & 9.25 & 0.144 & $2.0 \times 10^{-1}$ \\
\hline
\end{tabular}

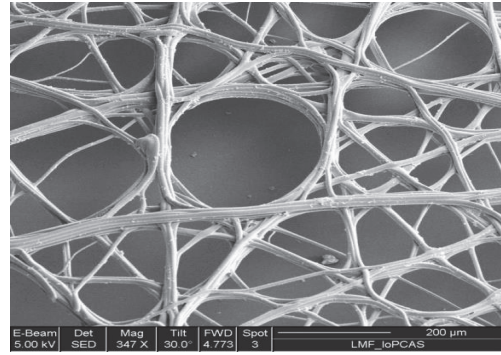

(a)

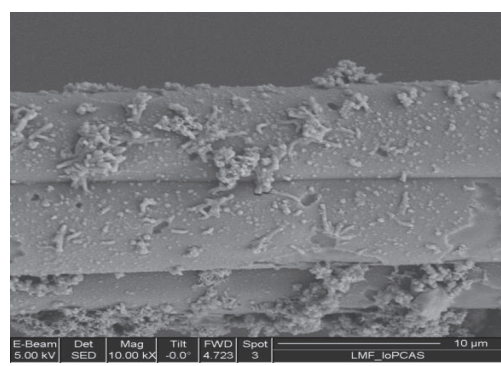

(c)

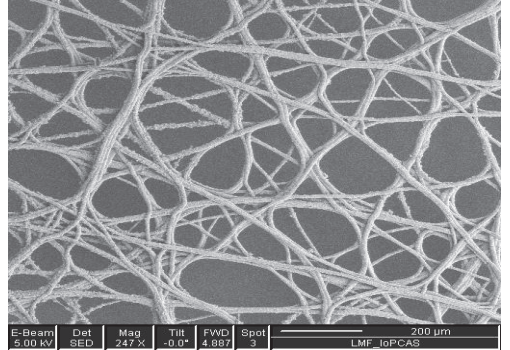

(b)

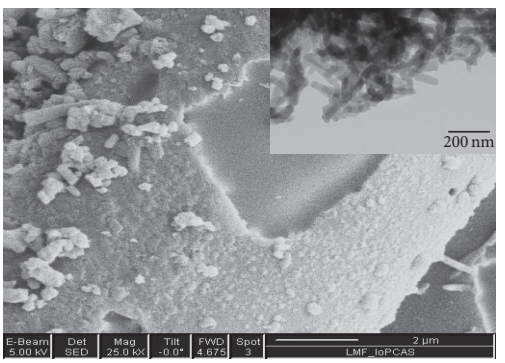

(d)

Figure 4: SEM images of (a) PMMA fibers, (b) PANI/PMMA composite fibers, and (c-d) enlarged SEM images of PANI/PMMA composite fibers. The inset is a TEM image of the PANI nanostructures on the surface of PANI/PMMA fibers.

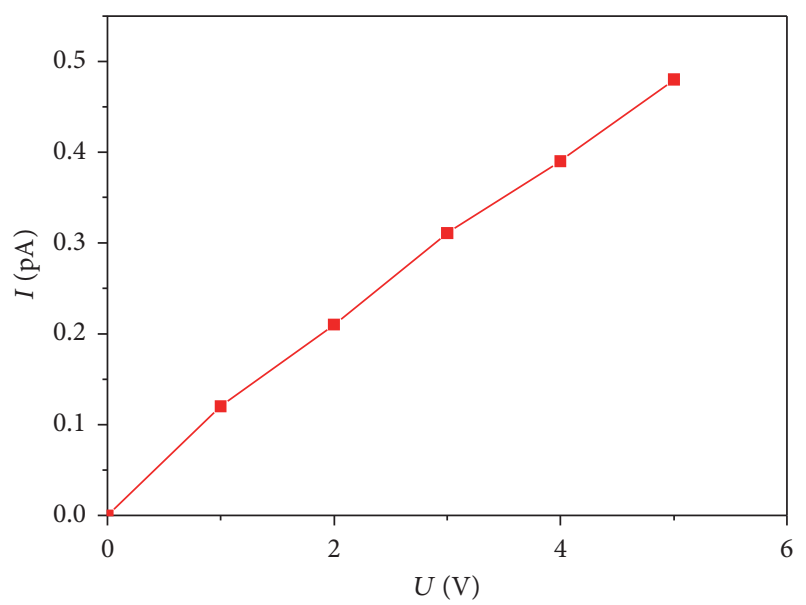

(a)

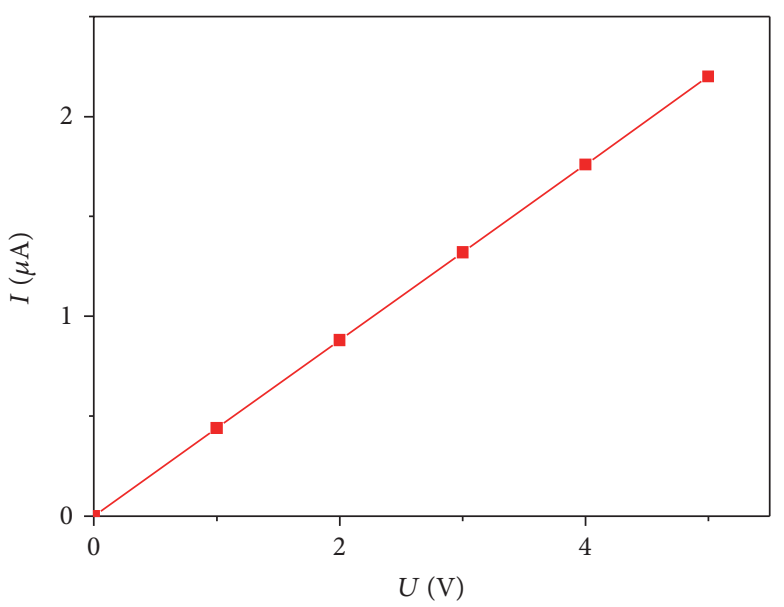

(b)

Figure 5: $I$ - $V$ characteristic curves of (a) as-spun PMMA fibers and (b) PANI/PMMA composite fibers at room temperature.

curvature was $0.91 \mathrm{~cm}^{-1}$. Figure $8(\mathrm{c})$ shows the resistance change with cycles of bending and relaxing. The bending curvature was $0.400 \mathrm{~cm}^{-1}$, and the test period was $10 \mathrm{~s}$ ( $3 \mathrm{~s}$ for bending and $7 \mathrm{~s}$ for relaxing). It can be seen that the sample shows a cyclic change in voltage with repeated cycles of bending and relaxing. The electrical resistance decreased as the sample was bended and then the resistance increased to the original value when the sample was relaxed. The decrease in resistance could be ascribed to the change of interfacial contacts among PANI fibers/grains. 


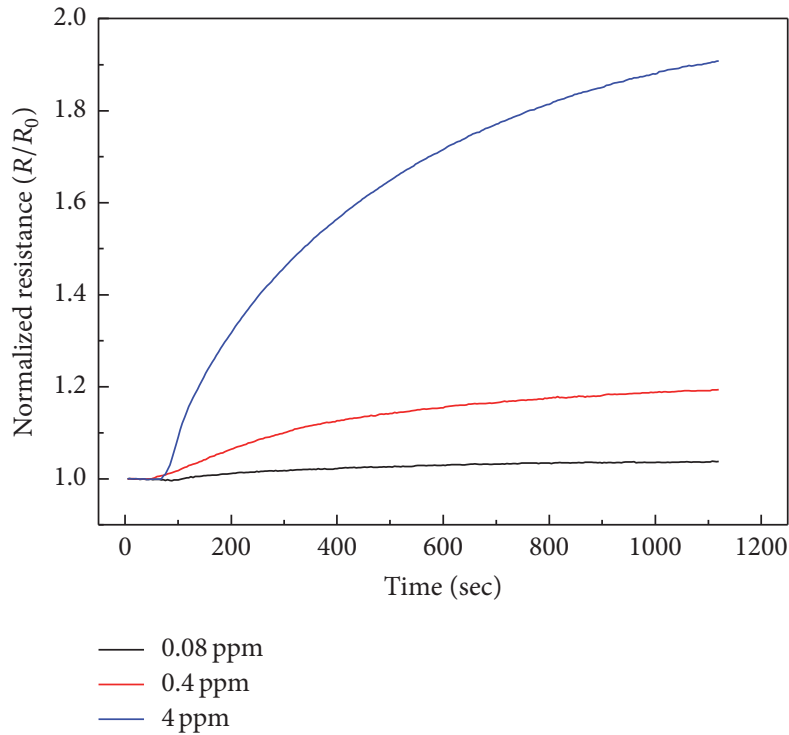

(a)

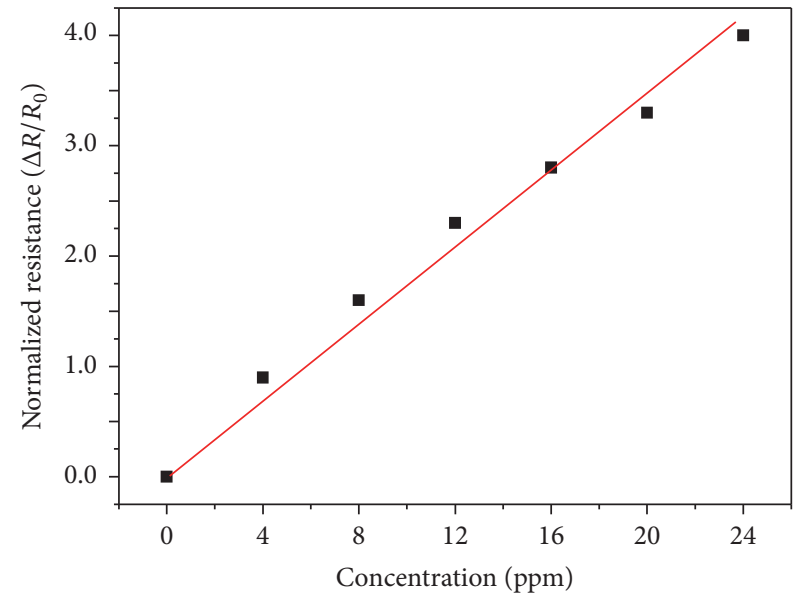

(b)

Figure 6: (a) Resistance variation of the PANI/PMMA fibers exposed to 0.08, 0.4, and 4 ppm ammonia gas at different time. (b) Sensitivity of the PANI/PMMA fibers at different ammonia concentration.

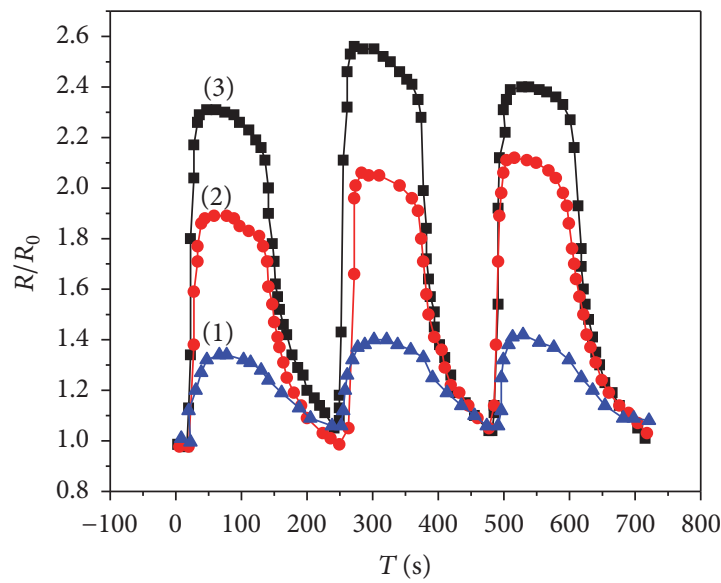

Figure 7: The reversible response curves of normalized electrical resistance to the $\mathrm{NH}_{3}$ concentration of $30 \mathrm{ppm}$ (1), $50 \mathrm{ppm}$ (2), and $80 \mathrm{ppm}$ (3).

\section{Conclusion}

In summary, PANI/PMMA composite fibers fabricated by in situ polymerization of PANI on the surface of electrospun PMMA fibers have good electrical conductivity and flexibility. The fabrication process is easy and low-cost and has the possibility of mass production. Both the gas and strain sensing properties of PANI/PMMA fibers are excellent. As a gas sensor, PANI/PMMA fibers have obvious response to low concentration of $\mathrm{NH}_{3}$ at ppb level and short response time to high concentration of $\mathrm{NH}_{3}$ at ppm level. As a strain sensor, its electrical resistance changes distinctly under bending, increases with increasing curvature, and shows a reversible change with repeated cycles of bending and relaxing. The results demonstrate that the PANI/PMMA fibers can be used in $\mathrm{NH}_{3}$ gas sensor, strain sensor, or flexible electronic device.

\section{Competing Interests}

The authors declare that there are no competing interests regarding the publication of this manuscript.

\section{Authors' Contributions}

Xian-Sheng Jia and Cheng-Chun Tang contributed to this work equally. 


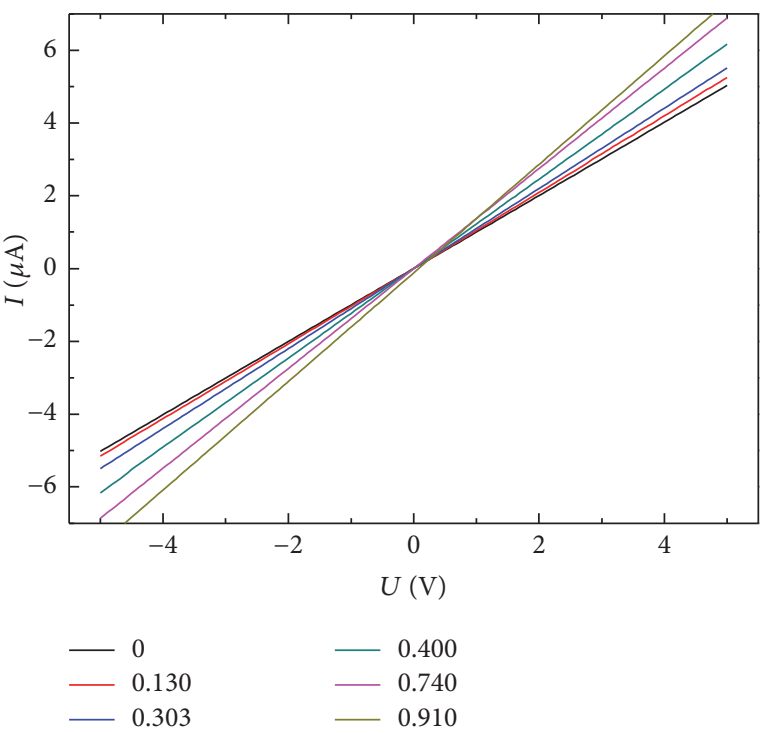

(a)

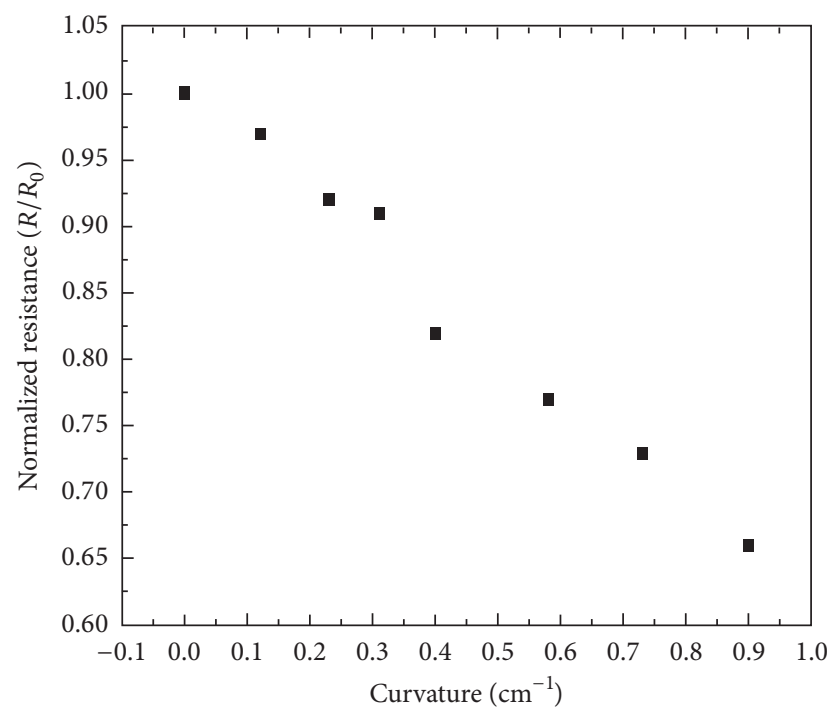

(b)

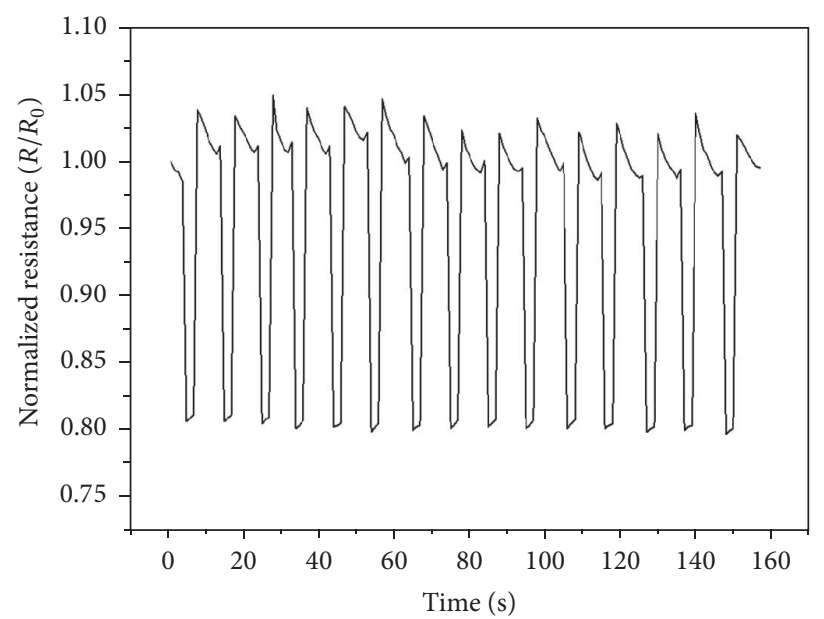

(c)

FIGURE 8: (a) $I-V$ characteristic curves of PANI/PMMA fibrous mat at different curvatures, (b) normalized resistance changes $\left(R / R_{0}\right)$ of PANI/PMMA sensor at different curvatures, and (c) its resistance changes with cycles of bending and relaxing (the bending curvature $\delta$ was $\left.0.400 \mathrm{~cm}^{-1}\right)$

\section{Acknowledgments}

This work was supported by the National Natural Science Foundation of China (51373082, 51673103, 91323304, 51272278, and 61390503) and the Taishan Scholars Programme of Shandong Province, China (ts20120528).

\section{References}

[1] G. Zheng, Q. Zhang, J. J. Cha et al., "Amphiphilic surface modification of hollow carbon nanofibers for improved cycle life of lithium sulfur batteries," Nano Letters, vol. 13, no. 3, pp. 1265-1270, 2013.

[2] W.-H. Ryu, T.-H. Yoon, S. H. Song, S. Jeon, Y.-J. Park, and I.-D. $\mathrm{Kim}$, "Bifunctional composite catalysts using $\mathrm{Co}_{3} \mathrm{O}_{4}$ nanofibers immobilized on nonoxidized graphene nanoflakes for highcapacity and long-cycle Li-O $\mathrm{O}_{2}$ batteries," Nano Letters, vol. 13, no. 9, pp. 4190-4197, 2013.
[3] G. Zhang and X. W. Lou, "Controlled growth of $\mathrm{NiCo}_{2} \mathrm{O}_{4}$ nanorods and ultrathin nanosheets on carbon nanofibers for highperformance supercapacitors," Scientific Reports, vol. 3, article 1470, 6 pages, 2013.

[4] F. Yang, R. Murugan, S. Wang, and S. Ramakrishna, "Electrospinning of nano/micro scale poly(l-lactic acid) aligned fibers and their potential in neural tissue engineering," Biomaterials, vol. 26, no. 15, pp. 2603-2610, 2005.

[5] X. Hu, S. Liu, G. Zhou, Y. Huang, Z. Xie, and X. Jing, "Electrospinning of polymeric nanofibers for drug delivery applications," Journal of Controlled Release, vol. 185, no. 1, pp. 12-21, 2014.

[6] A. Nikfarjam and N. Salehifar, "Improvement in gas-sensing properties of $\mathrm{TiO}_{2}$ nanofiber sensor by UV irradiation," Sensors and Actuators B: Chemical, vol. 211, pp. 146-156, 2015.

[7] B. Sun, Y.-Z. Long, Z.-J. Chen et al., "Recent advances in flexible and stretchable electronic devices via electrospinning," Journal of Materials Chemistry C, vol. 2, no. 7, pp. 1209-1219, 2014. 
[8] Z.-M. Huang, Y.-Z. Zhang, M. Kotaki, and S. Ramakrishna, "A review on polymer nanofibers by electrospinning and their applications in nanocomposites," Composites Science and Technology, vol. 63, no. 15, pp. 2223-2253, 2003.

[9] H. Fong, I. Chun, and D. H. Reneker, "Beaded nanofibers formed during electrospinning," Polymer, vol. 40, no. 16, pp. 4585-4592, 1999.

[10] S. Li, Y. Zhao, C. Wang, D. Li, and K. Gao, "Fabrication and characterization unique ribbon-like porous $\mathrm{Ag} / \mathrm{LaFeO}_{3}$ nanobelts photocatalyst via electrospinning," Materials Letters, vol. 170, pp. 122-125, 2016.

[11] J. Wu, N. Wang, L. Wang, H. Dong, Y. Zhao, and L. Jiang, "Electrospun porous structure fibrous film with high oil adsorption capacity," ACS Applied Materials and Interfaces, vol. 4, no. 6, pp. 3207-3212, 2012.

[12] X. Wang, H. Q. Fan, and P. R. Ren, "Electrospinning derived hollow $\mathrm{SnO}_{2}$ microtubes with highly photocatalytic property," Catalysis Communications, vol. 31, pp. 37-41, 2013.

[13] D. Li and Y. Xia, "Direct fabrication of composite and ceramic hollow nanofibers by electrospinning," Nano Letters, vol. 4, no. 5, pp. 933-938, 2004.

[14] M. M. Arafat, B. Dinan, S. A. Akbar, and A. S. M. A. Haseeb, "Gas sensors based on one dimensional nanostructured metaloxides: a review," Sensors, vol. 12, no. 6, pp. 7207-7258, 2012.

[15] B. J. Munro, T. E. Campbell, G. G. Wallace, and J. R. Steele, "The intelligent knee sleeve: a wearable biofeedback device," Sensors and Actuators, B: Chemical, vol. 131, no. 2, pp. 541-547, 2008.

[16] C. Cochrane, V. Koncar, M. Lewandowski, and C. Dufour, "Design and development of a flexible strain sensor for textile structures based on a conductive polymer composite," Sensors, vol. 7, no. 4, pp. 473-492, 2007.

[17] L. Maiolo, A. Pecora, F. Maita et al., "Flexible sensing systems based on polysilicon thin film transistors technology," Sensors and Actuators, B: Chemical, vol. 179, pp. 114-124, 2013.

[18] F. Martinez, G. Obieta, I. Uribe, T. Sikora, and E. Ochoteco, "Polymer-based flexible strain sensor," Procedia Chemistry, vol. 1, pp. 915-918, 2009.

[19] Y. Long, Z. Chen, N. Wang et al., "Electrical conductivity of a single conducting polyaniline nanotube," Applied Physics Letters, vol. 83, no. 9, pp. 1863-1865, 2003.

[20] R. Huang, Y. Z. Long, C. C. Tang, and H. D. Zhang, "Fabrication of nano-branched coaxial polyaniline / polyvinylidene fluoride fibers via electrospinning for strain sensor," Advanced Materials Research, vol. 853, pp. 79-82, 2014.

[21] N. Muthukumar, G. Thilagavathi, and T. Kannaian, "Polyaniline-coated nylon lycra fabrics for strain sensor and electromagnetic interference shielding applications," High Performance Polymers, vol. 27, no. 1, pp. 105-111, 2015.

[22] V. Talwar, O. Singh, and R. C. Singh, " $\mathrm{ZnO}$ assisted polyaniline nanofibers and its application as ammonia gas sensor," Sensors and Actuators, B: Chemical, vol. 191, pp. 276-282, 2014.

[23] J. Qi, X. Xu, X. Liu, and K. T. Lau, "Fabrication of textile based conductometric polyaniline gas sensor," Sensors and Actuators, B: Chemical, vol. 202, pp. 732-740, 2014.

[24] Y. Zhang and G. C. Rutledge, "Electrical conductivity of electrospun polyaniline and polyaniline-blend fibers and mats," Macromolecules, vol. 45, no. 10, pp. 4238-4246, 2012.

[25] J. H. Yu, S. V. Fridrikh, and G. C. Rutledge, "The role of elasticity in the formation of electrospun fibers," Polymer, vol. 47, no. 13, pp. 4789-4797, 2006.

[26] V. J. Babu, D. V. B. Murthy, V. Subramanian, V. R. K. Murthy, T. S. Natarajan, and S. Ramakrishna, "Microwave Hall mobility and electrical properties of electrospun polymer nanofibers," Journal of Applied Physics, vol. 109, no. 7, Article ID 074306, 2011.

[27] J. B. Veluru, K. K. Satheesh, D. C. Trivedi, M. V. Ramakrishna, and N. T. Srinivasan, "Electrical properties of electrospun fibers of PANI-PMMA composites," Journal of Engineered Fibers and Fabrics, vol. 2, pp. 25-31, 2007.

[28] G.-F. Yu, X. Yan, M. Yu et al., "Patterned, highly stretchable and conductive nanofibrous PANI/PVDF strain sensors based on electrospinning and in situ polymerization," Nanoscale, vol. 8, no. 5, pp. 2944-2950, 2016.

[29] A. Lanata and E. P. Scilingo, "Smart textiles: technology and wireless system network applications," in Autonomous Sensor Networks, pp. 127-158, Springer, Berlin, Germany, 2012.

[30] X. B. Yan, Z. J. Han, Y. Yang, and B. K. Tay, " $\mathrm{NO}_{2}$ gas sensing with polyaniline nanofibers synthesized by a facile aqueous/organic interfacial polymerization," Sensors and Actuators, B: Chemical, vol. 123, no. 1, pp. 107-113, 2007.

[31] H.-D. Zhang, C.-C. Tang, Y.-Z. Long et al., "High-sensitivity gas sensors based on arranged polyaniline/PMMA composite fibers," Sensors and Actuators A: Physical, vol. 219, pp. 123-127, 2014. 

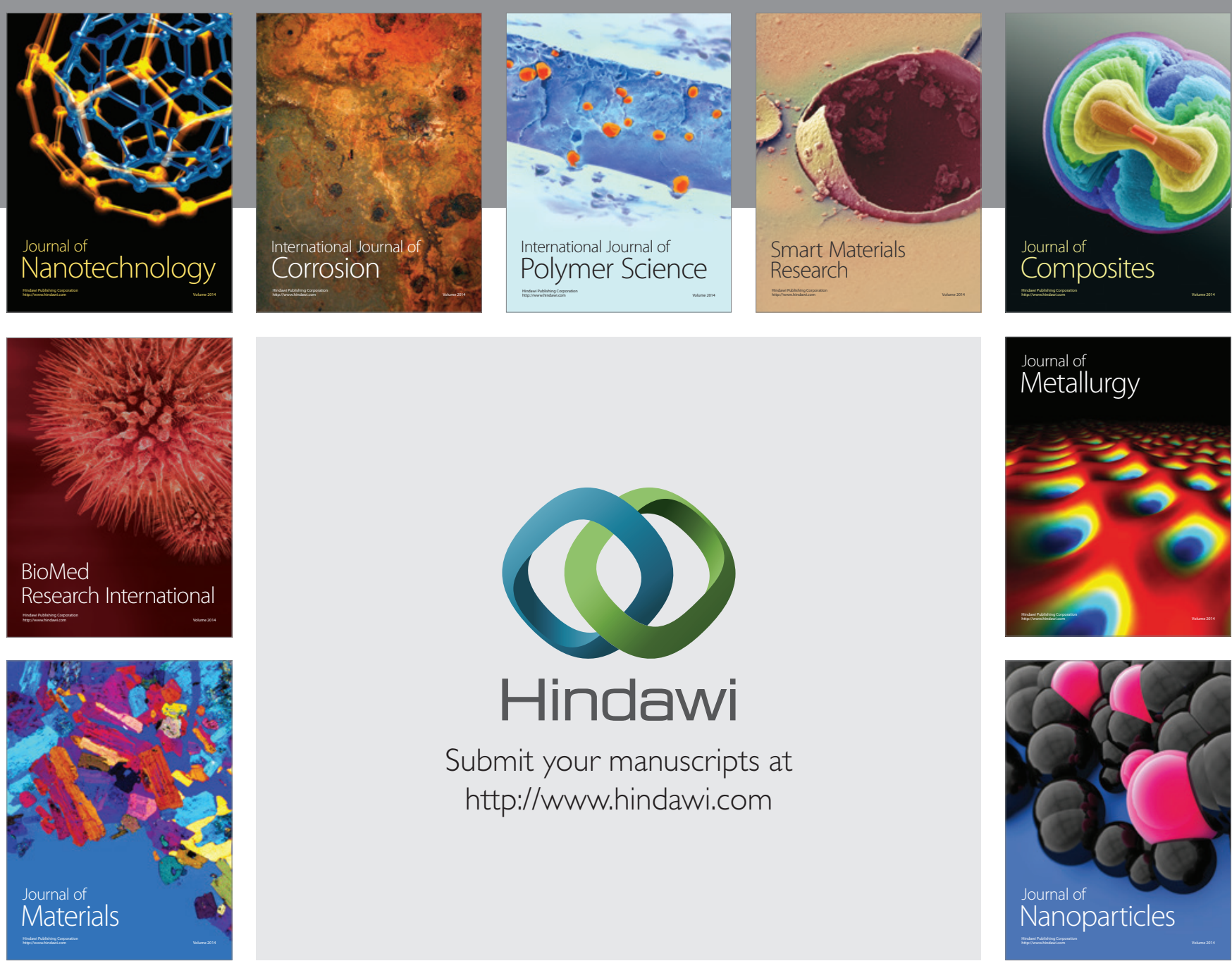

\section{Hindawi}

Submit your manuscripts at

http://www.hindawi.com

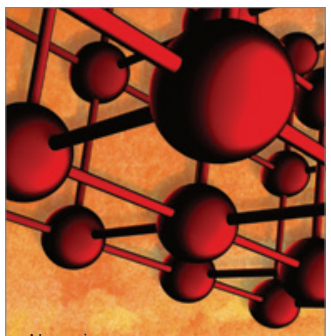

Materials Science and Engineering
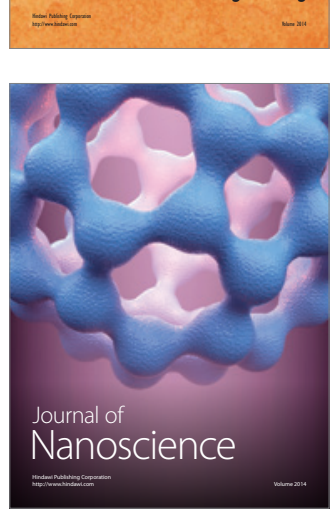
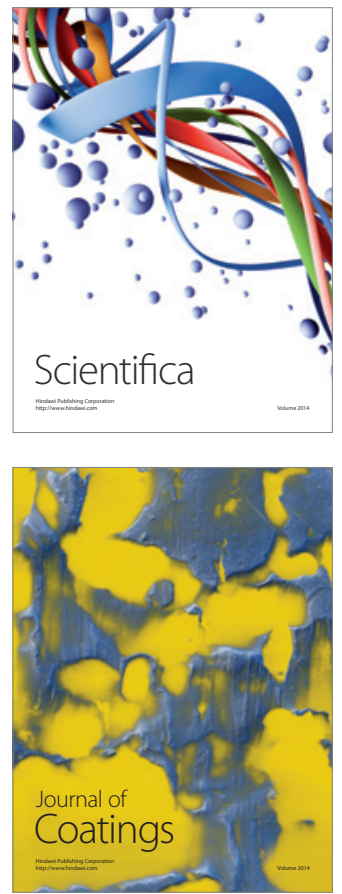
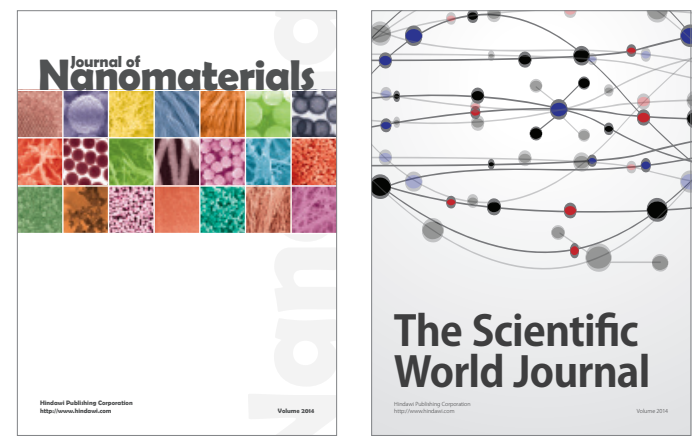

The Scientific World Journal
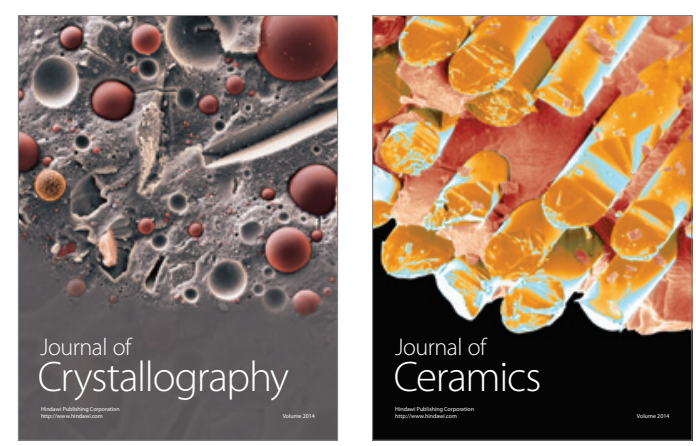
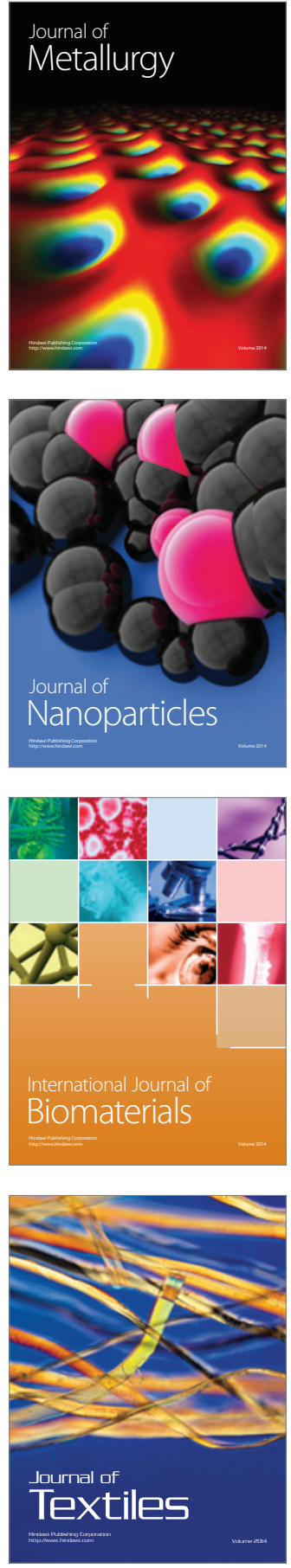